\title{
Data Level Inference Detection in Database Systems*
}

\author{
Raymond W. Yip and Karl N. Levitt \\ Department of Computer Science \\ University of California, Davis \\ One Shields Avenue, Davis CA 95616
}

\begin{abstract}
Existing work on inference detection for database systems mainly employ functional dependencies in the database schema to detect inferences. It has been noticed that analyzing the data stored in the database may help to detect more inferences. In this paper, we describe our effort in developing a data level inference detection system. We have identified five inference rules that a user can use to perform inferences. They are 'subsume', 'unique characteristic', 'overlapping', 'complementary', and 'functional dependency' inference rules. The existence of these inference rules confirms the inadequacy of detecting inferences using just functional dependencies. The rules can be applied any number of times and in any order. These inference rules are sound. They are not necessarily complete, although we have no example that demonstrates incompleteness. We employ a rule based approach so that future inference rules can be incorporated into the detection system. We have developed a prototype of the inference detection system using Perl on a Sun SPARC 20 workstation. The preliminary results show that on average it takes seconds to process a query for a database with thousands of records. Thus, our approach to inference detection is best performed off-line, and would be most useful to detect subtle inference attacks.
\end{abstract}

\section{Introduction}

Inference is a method to subvert access control in database systems. An inference occurs when a user is able to infer some data without directly accessing them. In multilevel database systems, early work on inference detection used a graph to represent the

\footnotetext{
* Copyright 1998 IEEE. Published in Proceedings, 1998 IEEE Computer Security Foundations Workshop, Rockport, Massachusetts, June, 1998.
}

functional dependencies among the attributes in the database schema. An inference occurs when there are two or more paths among the attributes, and the paths are labeled at different classification levels $[6,2,12]$. The inference path is eliminated by upgrading some attributes along the path [15, 13]. Lunt [9] points out that some inference problems can be avoided by redesigning the database schema, and classifying the attributes properly. However, redesigning the database schema results in data duplication which leads to update anomalies. It also requires modifications to the existing application programs. There is also work on incorporating external knowledge into the inference detection systems $[18,7,16,17,3]$. More recently, researchers suggest using data of the database to generate a richer set of functional dependencies for inference detection. Hinke et al. use cardinality associations to discover potential inference paths [8]. Hale et al. incorporate imprecise and fuzzy database relations into their inference detection system [5]. However, existing efforts still simply employ functional dependencies to detect inferences. As noted by SRI researchers, monitoring user activities may lead to detecting more inferences [14]. By data level inference detection, we mean the system detects inferences by considering the data in the database, as opposed to the database schema only.

Inferences can also occur in discretionary access control systems where users are explicitly granted access rights to access data (as in System R). It is not an obvious task to grant users the exact amount of access rights they need. In some cases, users are simply granted more access rights than they need in order not to hinder their work. To ensure the users do not misuse the database, we need to monitor their accesses.

A simple way to monitor user accesses is to examine each user query, and reject any query that accesses sensitive data. However, it is possible for a user to use a series of unsuspicious queries to infer data in the database. Motro et al. address a similar problem, but 
their work focuses on detecting aggregation instead of inference attacks [11]. In the statistical database security community, various techniques have been proposed to protect individual records, for example, query-setsize control, cell suppression, and data perturbation [1]. However, these techniques are not suitable for detecting inferences using general purpose queries.

In this paper, we describe our effort in developing a data level inference detection system. It is a static inference detection system where inferences are performed with respect to a snapshot of the database. We have identified five inference rules that users can use to infer data: 'subsume', 'unique characteristic', 'overlapping', 'complementary', and 'functional dependency' inference rules. Users can apply these rules any number of times, and in any order to infer data. These inference rules are sound but not necessarily complete. Although we have no example that demonstrates incompleteness, more research effort is needed to determine if they are complete. We employ a rule based approach so that when a new inference rule is discovered, it can be incorporated into the inference detection system. The existence of these inference rules confirms the inadequacy of functional-dependency based inference detection schemes. We have developed a prototype of the inference detection system to study its performance. The preliminary results show that on average the system takes a few seconds to process a query that returns hundreds of records from a database of ten thousand records. Thus, our approach to inference detection is best performed off-line, and would be most useful to detect subtle inference attacks.

This paper is organized as follows. In Section 2, we provide the intuition behind the five inference rules. In Section 3, we introduce the notations used in this paper. In Section 4, we present the five inference rules. In Section 5, we describe our implementation and the preliminary results. We give our conclusions in Section 6.

\section{Overview of the Inference Rules}

In this section, we provide the intuition behind the five inference rules. The goal of our inference detection system is to detect if a user can indirectly access data using two or more queries. In particular, the system determines if the user can infer the return tuples from different queries corresponding to the same tuple in the database.

The result of each user query is a set of return tuples 1. The user cannot identify each return tuple unless

\footnotetext{
${ }^{1}$ Unless otherwise stated, a set of return tuples is indeed a
}

the primary key of the tuple is also returned. However, a certain group of attribute values of a tuple may uniquely identify the tuple. The unique identification rule handles this situation. Another way to identify a return tuple is to compare it with other return tuples that have already been identified.

There are two possible relationships between two sets of return tuples. One possibility is that for each return tuple $t_{1}$ of a query, there is a return tuple $t_{2}$ of the other query, such that $t_{1}$ and $t_{2}$ correspond to the same tuple in the database. The subsume inference rule handles this case. Another possibility is that only some return tuples of a query correspond to some return tuples of another query. The overlapping inference rule identifies the corresponding return tuples that are common to both queries. The complementary inference rule identifies the corresponding return tuples by taking the "difference" between two sets of return tuples. The functional dependency inference rule is introduced to simulate the schema level inference detection scheme.

Once the corresponding return tuples between two queries are identified, the user can generate inferred queries. The user knows the return tuples of an inferred query without directly issuing it to the database. For example, the user can infer a new query with returns tuples "common" to both queries, or a new query that returns tuples from one query but not from another query. The user can also combine several queries into a single query. We will discuss the effect of applying inference rules to unions of queries. Essentially, the five inference rules cover the set intersection, difference and union relationships between two sets of return tuples.

When the user issues a query, the inference detection system compares it with previously issued queries and inferred queries, and applies inference rules when appropriate. An occurrence of inference will result in either the modifications of the existing queries (for example, combining two corresponding return tuples), or the generation of new inferred queries. These may trigger further applications of the inference rules. Hence, the inference rules are applied repeatedly until there is no new inference occurs. This is a terminating process as the number of inferences that can occur is bounded by the size of the database. When two users are suspected of cooperating in performing inference, we can run the inference detection system against their combined set of queries.

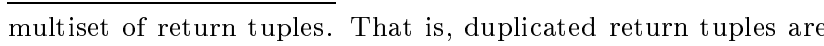
not removed 


\section{Preliminaries and Notation}

We consider inference detection in a relational database with a single table. A database with multiple tables can be transformed into a universal relation [10]. We assume that the only way the user can learn about the data in the database is by issuing queries to it. That is the user does not rely on real-world knowledge to perform any inference. Such knowledge might be added to the database as 'catalytic relation' as suggested in [6].

$A_{i}$ denotes an attribute in the table, and $a_{i}$ denotes an attribute value from the domain of $A_{i} . t\left[A_{i}\right]$ denotes the attribute value of a single tuple $t$ over the attribute $A_{i}$. A query is represented by a 2-tuple: (attribute-set, selection-criterion), where attribute-set is the set of attributes projected by the query, and selection-criterion is the logical expression that is satisfied by each return tuple of the query. No aggregation function (for example, maximum and average) is allowed in the attributeset. In general, $Q_{i}$ refers to the query $\left\{A S_{i} ; S C_{i}\right\} .\left|Q_{i}\right|$ denotes the number of return tuples of $Q_{i} .\left\{Q_{i}\right\}$ denotes the set of return tuples of $Q_{i}$. For each query $Q_{i}$, $A S_{i}$ is expanded with an attribute $A_{i}$ when ' $A_{i}=a_{i}$ ', appears in $S C i$ as a conjunct. A query $Q$ is a 'partial query' if the user can determine $|Q|$, but not all return tuples of $Q$. ' $\cap$ ', ' $\cup$ ', and ' $\backslash$ ' stand for the set intersection, union, and difference operation respectively.

Now, we introduce several notions that are used throughout this paper.

Definition 1 A tuple $t$ over a set of attributes $A S$ 'satisfies' a logical expression $E$ if $E$ is evaluated to true when each occurrence of $A_{i}$ in $E$ is instantiated with $t\left[A_{i}\right]$, for all $A_{i}$ in $A S$. $t$ 'contradicts' with $E$ if $E$ is evaluated to false.

For example, the tuple $(35,60 \mathrm{~K})$ that is projected over (Age, Salary) satisfies $E=($ Age $>30 \wedge$ Salary $<70 K)$; while the tuple $(25,50 K)$ projected over the same set of attributes contradicts with $E$. The tuple (45K, Manager) projected over (Salary, Job) neither satisfies nor contradicts $E$. This is because after the instantiation, $E$ becomes (Salary $<70 \mathrm{~K}$ ) whose truth value is undetermined.

Definition 2 Given two queries, $Q_{1}$ and $Q_{2}$, we say that $Q_{1}$ is 'subsumed' by $Q_{2}$, denoted as $Q_{1} \sqsubset Q_{2}$, iff

$$
\begin{aligned}
& \text { 1. } S C_{1} \rightarrow S C_{2} \text {; or } \\
& \text { 2. for each tuple } t_{1} \text { in }\left\{Q_{1}\right\}, t_{1} \text { satisfies } S C_{2} \text {. }
\end{aligned}
$$

where $\rightarrow$ is the logical implication. ' $\square$ ' is a reflexive, anti-symmetric, and transitive relation. A return tuple $t_{1}$ 'relates' to another return tuple $t_{2}$ if the two tuples are selected from the same tuple in the database. Hence, $Q_{1} \sqsubset Q_{2}$ implies that for each return tuple $t_{1}$ of $Q_{1}$, there is a return tuple $t_{2}$ of $Q_{2}$, such that $t_{1}$ relates to $t_{2}$.

When evaluating a logical implication, we need to consider the integrity constraints that hold in the database. Consider the following implication,

(age $>18 \wedge$ age $<35) \rightarrow($ age $>20 \wedge$ age $<50)$, which is false. Suppose the youngest person in the database is 22 years old. By adding this constraint to both sides of the implication, it becomes,

$(($ age $>18 \wedge$ age $<35) \wedge($ age $\geq 22)) \rightarrow$

$(($ age $>20 \wedge$ age $<50) \wedge($ age $\geq 22)) \equiv$

(age $\geq 22 \wedge$ age $<35) \rightarrow$

(age $\geq 22 \wedge$ age $<50$ ),

which is true.

Now, we introduce the notion of 'indistinguishable'.

Definition 3 A return tuple 11 of $Q 1$ is 'indistinguishable' from a return tuple t2 of $Q 2$ iff

1. for all $A_{i}$ in $\left(A S_{1} \cap A S_{2}\right), t_{1}\left[A_{i}\right]=t_{2}\left[A_{i}\right]$;

2. $t_{1}$ does not contradict with $S C_{2}$; and

3. $t_{2}$ does not contradict with $S C_{1}$.

$t_{1}$ is 'distinguishable' from $t_{2}$ if $t_{1}$ is not indistinguishable from $t_{2}$.

Intuitively, $t_{1}$ is indistinguishable from $t_{2}$ if it is not possible to conclude that $t_{1}$ and $t_{2}$ are selected from two different tuples in the database. Two tuples that relate to each other are indistinguishable from each other, while two tuples that are indistinguishable from each other does not imply that they relate to each other.

Our system only detects inferences of the "right instances" of the data. Consider the following table,

\begin{tabular}{|c|c|c|c|}
\hline Name & Job & Age & Salary \\
\hline \hline John & Engineer & 29 & $60 \mathrm{~K}$ \\
\hline Paul & Engineer & 31 & $60 \mathrm{~K}$ \\
\hline
\end{tabular}

Suppose the user knows that John is an engineer, and that there is an engineer who is 31 years old and earns $60 \mathrm{~K}$. A naive user may conclude that John earns $60 \mathrm{~K}$, assuming that John's age is 31. Although the user correctly infers the salary of John, this is not the right instance of the salary of John. In fact, when the user learns that John is indeed 29, the user will revoke this inference. In our model, a skeptical user will not make such hasty inferences. 


\begin{tabular}{|c|c|c|c|c|c|}
\hline Name & Job & Age & Salary & Department & Office \\
\hline \hline Alice & Manager & 35 & $60 \mathrm{~K}$ & Marketing & 2nd Floor \\
\hline Bob & Secretary & 35 & $45 \mathrm{~K}$ & Marketing & 2nd Floor \\
\hline Charles & Secretary & 40 & $40 \mathrm{~K}$ & Production & 1st Floor \\
\hline Denise & Manager & 45 & $65 \mathrm{~K}$ & Sales & 2nd Floor \\
\hline
\end{tabular}

Figure 1. Sample database.

\section{Inference Rules}

In this section, we describe the five inference rules. We illustrate the inference rules using the sample database as shown in Figure 1. The user is allowed to access all data in the database. However, it is suspicious if the user can infer the salaries of employees. We assume the the security policy is to determine if the user infer the associations between 'Name' and 'Salary'. In general, the policy can specify detecting inferences of any association among the attributes. Unless otherwise stated, all queries appear in the inference rules are not partial queries.

\subsection{Subsume Inference}

In this section, we describe the use of the ' $\square$ ' relations to perform inferences.

Inference Rule 1 (Subsume) Given two queries $Q_{1}$ and $Q_{2}$, such that $Q_{1} \sqsubset Q_{2}$.

SI1 If there is an attribute $A$ in $\left(A S_{2} \backslash A S_{1}\right)$, such that all return tuples of $Q_{2}$ take the same attribute value a over $A$, then for each return tuple $t_{1}$ of $Q_{1}$, $t_{1}[A]=a . Q_{1}$ may be a partial query.

SI2 If there is a return tuple $t_{1}$ of $Q_{1}$ that is indistinguishable from one and only one return tuple $t_{2}$ of $Q_{2}$, then $t_{1}$ relates to $t_{2} . Q_{1}$ may be a partial query.

SI3 Let $S$ be the set of return tuples of $Q_{2}$ that are distinguishable from the return tuples of $Q_{1}$. If $|S|$ $=\left(\left|Q_{2}\right|-\left|Q_{1}\right|\right)$, then two inferred queries are generated: $\left(A S_{2} ; S C_{2} \wedge \neg S C_{1}\right)$ with $S$ as the set of return tuples, and $\left(A S_{2} ; S C_{2} \wedge S C_{1}\right)$ with $\left(\left\{Q_{2}\right\} \backslash\right.$ $S)$ as the set of return tuples. If $|S|<\left(\left|Q_{2}\right|-\left|Q_{1}\right|\right)$, then an inferred partial query is generated: $\left(A S_{2}\right.$; $S C_{2} \wedge \neg S C_{1}$ ) with $S$ as the partial set of return tuples.

$Q_{1} \sqsubset Q_{2}$ implies that for each return tuple $t_{1}$ of $Q_{1}$, there is a return tuple $t_{2}$ of $Q_{2}$, such that $t_{1}$ relates to $t_{2}$. SI1 says that when all return tuples of $Q_{2}$ share a common attribute value, say $a$, over an attribute $A$, the user can infer that each return tuple of $Q_{1}$ also take the attribute value $a$ over the attribute $A$. For example, consider the following two queries:

$Q_{1}=($ Age; Name $=$ 'Alice'), and

$Q_{2}=$ (Department; Age < 40).

$Q_{1}$ returns a single tuple (35) which says that Alice is 35 years old. $Q_{2}$ returns two tuples ('Marketing') and ('Marketing') which shows that all employees at the age less than 40 work in the Marketing department. By SI1, Alice works in the Marketing department.

SI2 says that if $t_{2}$ is the only return tuple of $Q_{2}$ that is indistinguishable from a return tuple $t_{1}$ of $Q_{1}$, then $t_{1}$ relates to $t_{2}$. Consider the following two queries:

$Q_{3}=($ Age; Name $=$ 'Charles'), and

$Q_{4}=($ Age, Salary; Age $\geq 40)$.

$Q_{3}$ returns a single tuple $t_{3}=(40)$ which says that Charles is 40 years old. $Q_{4}$ returns two tuples (40, $40 K)$ and $(45,65 K)$ which says that there are only two employees who are at the age greater than or equal to 40. As $Q_{3} \sqsubset Q_{4}$ (since (40) satisfies $\left.S C_{4}\right)$ and (40, $40 K)$ is the only return tuple of $Q_{4}$ that is indistinguishable from $t_{3}$, by SI2, Charles earns $40 \mathrm{~K}$.

$S I 3$ says that if a user identifies all the return tuples of $Q_{2}$ that relate to the return tuples of $Q_{1}$, then the user can infer these two queries: $\left(A S_{2} ; S C_{1} \wedge S C_{2}\right)$ which includes return tuples of $Q_{2}$ that relate to the return tuples of $Q 1$, and $\left(A S_{2} ; S C_{2} \wedge \neg S C_{1}\right)$ which includes return tuples of $Q_{2}$ that do not relate to the return tuples of $Q_{1}$. Continue from the above example on $Q_{3}$ and $Q_{4}$, after the application of SI2, we generate the following two inferred queries:

$Q_{21}=($ Age, Salary; Name $=$ 'Charles' $\wedge$ Age $\geq 40)$, and

$Q_{22}=($ Age, Salary; Name $\neq$ 'Charles' $\wedge$ Age $\geq 40)$. $Q_{21}$ returns a single tuple $(40,40 K)$, and $Q_{22}$ returns a single tuple $(45,65 K)$. The two inferred queries together contains more information than $Q_{2}$. For example, $Q_{22}$ says that the employee who is at the age of 45 and earns $65 \mathrm{~K}$ must be someone other than Charles.

\subsection{Unique Characteristic Inference}

A unique characteristic is defined as follows, 


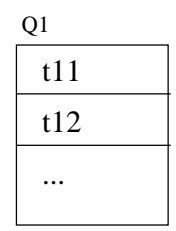

(a)

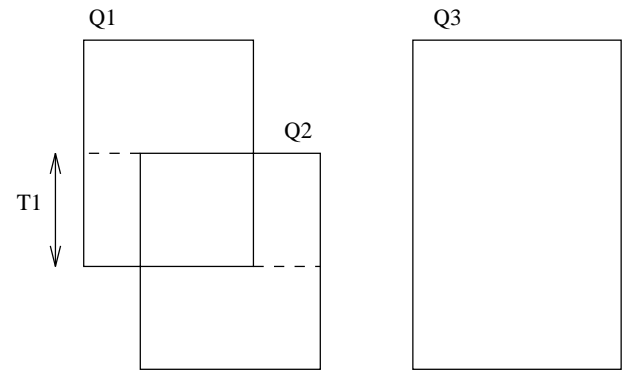

(b)

Figure 2. Examples on overlapping inference.

Definition 4 A logical expression $E$ is a unique characteristic of a tuple $t$ iff $t$ is the only tuple in the database that satisfies $E$.

For example, if Alice is the only manager at the age of 35 , then ( $J o b=$ 'Manager' $\wedge$ Age $=35)$ is the unique characteristic of Alice in the database.

\section{Inference Rule 2 (Unique Characteristic)}

Given a tuple $t_{1}$ with unique characteristic $C_{1}$ in a database $D$, and another tuple $t_{2}$ with unique characteristic $C_{2}$ in D. If $C_{1} \rightarrow C_{2}, C_{2} \rightarrow C_{1}$, or $C_{1} \leftrightarrow C_{2}$ (that is $C_{1} \rightarrow C_{2}$ and $C_{2} \rightarrow C_{1}$ ), then $t_{1}$ relates to $t_{2}$ in $D$.

For example, the query

(Salary; Job $=$ 'Manager' $\wedge$ Age $\leq 40)$

returns a single tuple $(60 K)$. This query together with the above unique characteristic of Alice implies Alice earns $60 \mathrm{~K}$. Unique characteristic inference is a special case of the subsume inference. Suppose $\left(A S_{1} ; U C_{1}\right)$ returns a single tuple $t_{1}$, and $\left(A S_{2} ; U C_{2}\right)$ returns a single tuple $t_{2}$. Then, $U C_{1}$ is the unique characteristic of $t_{1}$, and $U C_{2}$ is the unique characteristic of $t_{2}$. If $U C_{1} \rightarrow U C_{2}, U C_{2} \rightarrow U C_{1}$, or $U C_{1} \leftrightarrow U C_{2}$ holds, then by $S I 2, t_{1}$ relates to $t_{2}$.

If all inferred queries are identified, unique characteristics are determined as follows,

1. if $Q_{i}$ returns all but one tuple $t$ in the database, then the unique characteristic of $t$ is $\left(\neg S C_{i}\right)$.

2. if $Q_{i}$ and $Q_{j}$ have only one overlapping return tuple $t$, then $t$ has the unique characteristic $\left(S C_{i} \wedge\right.$ $S C_{j}$ ).

3. if $Q_{i}$ returns one more tuple $t$ than $Q j$, then the unique characteristic of $t$ is $\left(S C_{i} \wedge \neg S C_{j}\right)$. where both $Q_{i}$ and $Q_{j}$ are not partial queries. The determination of the overlapping tuples between two queries is discussed in the Section 4.3.

\subsection{Overlapping Inference}

In this section, we describe the overlapping inference rule.

Inference Rule 3 (Overlapping) Given $n$ queries $Q_{1}, \ldots, Q_{n}$, where $n \geq 3$.

OI1 Let $S$ be the set of return tuples of $Q_{2}$ that are indistinguishable from the return tuples of $Q_{3}$. If $Q_{1} \sqsubset Q_{2}, Q_{1} \sqsubset Q_{3},|S|=\left|Q_{1}\right|$, and $t_{2}$ is the only return tuple of $Q_{2}$ that is indistinguishable from a return tuple $t_{3}$ of $Q_{3}$, then $t_{2}$ relates to $t_{3} . Q_{1}$ may be a partial query.

OI2 Let $Q S=\left\{Q_{2}, \ldots, Q_{n}\right\}$. Suppose for each $Q_{i}$ in $Q S, Q_{i} \sqsubset Q_{1}$, and the return tuples of $Q_{i}$ are indistinguishable from the return tuples of at most one other query in QS. Also, the total number of indistinguishable tuples in all queries in $Q S$ is equal to $\left(2 \times\left(\left|Q_{2}\right|+\ldots+\left|Q_{n}\right|-\left|Q_{1}\right|\right)\right)$. For any two queries $Q j$ and $Q k$ in $Q S$, if $t_{j}$ is the only return tuple of $Q_{j}$ that is indistinguishable from a return tuple $t_{k}$ of $Q_{k}$, then $t_{j}$ relates to $t_{k}$. $Q_{1}$ may be a partial query.

OI3 When all relating tuples between $Q_{i}$ and $Q_{j}$ are identified, three inferred queries are generated (possibly partial): $\left(A S_{i} ; S C_{i} \wedge \neg S C_{j}\right),\left(A S_{j}\right.$; $\left.S C_{j} \wedge \neg S C_{i}\right)$, and $\left(A S_{i} \cap A S_{j} ; S C_{i} \wedge S C_{j}\right)$.

Figure 2(a) illustrates OI1. Each rectangle represents a set of return tuples of a query. The rectangles are drawn in such a way that return tuples that are selected from the same tuple in the database are aligned 


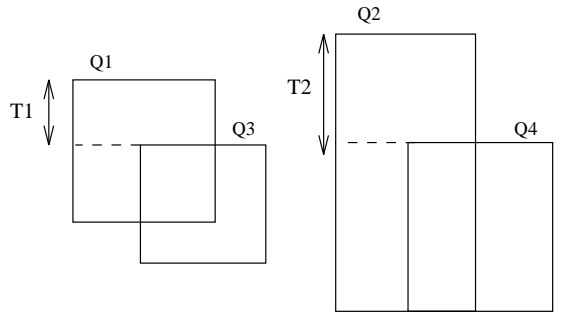

(a)

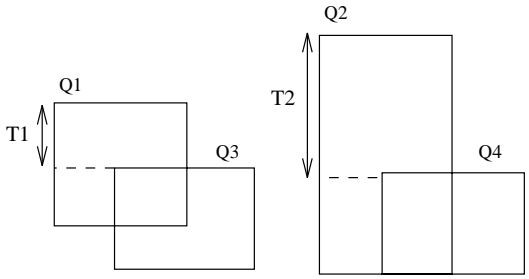

(b)

Figure 3. Examples on complementary inference.

horizontally. For example, $t_{11}, t_{21}$, and $t_{31}$ correspond to the same tuple in the database. Suppose $Q_{1} \sqsubset Q_{2}$ and $Q_{1} \sqsubset Q_{3}$, and the number of indistinguishable tuples between $Q_{2}$ and $Q_{3}$ equals $\left|Q_{1}\right|$. This implies that for each return tuple $t_{11}$ of $Q_{1}$, there is a return tuple $t_{21}$ of $Q_{2}$, and a return tuple $t_{31}$ of $Q_{3}$, such that $t_{11}$ relates to $t_{21}$, and $t_{11}$ relates to $t_{31}$; that is, $t_{21}$ relates to $t_{31}$. We further illustrate $O I 1$ by an example. Consider the following three queries,

$Q_{1}=($ Name; Job $=$ 'Manager' $\wedge$ Age $=35)$,

$Q_{2}=($ Salary; Job $=$ 'Manager'), and

$Q_{3}=($ Salary; Age $=35)$.

$Q_{1}$ returns a single tuple (Alice) which says that Alice is the only manager at the age of $35 . Q_{2}$ returns two tuples (60K) and (65K). $Q_{1}$ and $Q_{2}$ together implies that the salary of Alice is either $60 \mathrm{~K}$ or $65 \mathrm{~K} . Q_{3}$ returns two tuples (60K) and (45K). $Q_{1}$ and $Q_{3}$ together implies that the salary of Alice is either $60 \mathrm{~K}$ or $45 \mathrm{~K}$. As $Q_{1} \sqsubset Q_{2}$ and $Q_{1} \sqsubset Q_{3}$, and there is only one return tuple of $Q_{2}$ that is indistinguishable from return tuples of $Q_{3}$, namely the tuple $(60 \mathrm{~K})$. Hence, by $O I 1$, Alice earns $60 \mathrm{~K}$. When $Q_{1}$ implies three or more queries, OI1 is applied to two of them at a time.

Figure 2(b) illustrates OI2. Let $T_{1}$ be the set of return tuples of $Q_{1}$ that relate to return tuples of $Q_{2}$. $\left(\left|Q_{1}\right|+\left|Q_{2}\right|-\left|T_{1}\right|\right)$ is the number of tuples in both $Q_{1}$ and $Q_{2}$ that do not relate to one another. When $\left(\left|Q_{1}\right|+\left|Q_{2}\right|-\left|T_{1}\right|\right)=\left|Q_{3}\right|$, the user can infer that for each return tuple $t_{1}$ of $Q_{1}$ that is indistinguishable from a return tuple $t_{2}$ of $Q_{2}, t_{1}$ relates to $t_{2}$. As the tuples that are indistinguishable from each other appear in exactly two queries, the number of indistinguishable tuples equals $2 \times\left(\left|Q_{1}\right|+\left|Q_{2}\right|-\left|Q_{3}\right|\right)$. We further illustrate OI2 with the following three queries,

$$
\begin{aligned}
Q_{1}= & (\text { Salary; Department }=\text { 'Marketing' } \wedge \\
& \text { Office }=\text { '2nd Floor' }), \\
Q_{2}= & (\text { Salary; Job }=\text { 'Manager' } \wedge \\
& \text { Office }=\text { '2nd Floor' }), \text { and } \\
Q_{3}= & (\text { Name; Office }=\text { '2nd Floor' }) .
\end{aligned}
$$

$Q_{1}$ returns two tuples (60K) and (45K) which says that the two employees who work in the Marketing department on the 2 nd floor earn either $60 \mathrm{~K}$ or $45 \mathrm{~K} . Q_{2}$ returns two tuples $(60 K)$ and $(65 K)$ which says that the two managers who work on the 2 nd floor earn either $60 \mathrm{~K}$ or $65 \mathrm{~K} . Q_{3}$ returns three tuples (Alice), (Bob), and (Denise) which says that Alice, Bob and Denise all work on the 2 nd Floor. We have 1) $Q_{1} \sqsubset Q_{3}$, 2) $\left.Q_{2} \sqsubset Q_{3}, 3\right)$ there is only one return tuple of $Q_{1}$ that is indistinguishable from a return tuple of $Q_{2}$, namely the tuple $\{(60 K)\}$ (that is, the number of indistinguishable tuples in both $Q_{1}$ and $Q_{2}$ is 2), and 4) $2 \times\left(\left|Q_{1}\right|+\left|Q_{2}\right|-\left|Q_{3}\right|\right)=2 \times(2+2-3)=2$. By OI2, the tuple (60K) of $Q_{1}$ relates to the tuple (60K) of $Q_{2}$. That is, the user can infer that the marketing manager who works on the 2 nd floor earns $60 \mathrm{~K}$.

\subsection{Complementary Inference}

Complementary inference rule performs inferences by eliminating tuples that are not relating to one another.

Inference Rule 4 (Complementary Inference) Given four queries, $Q_{1}, Q_{2}, Q_{3}$, and $Q_{4}$, where $Q_{1}$ $\sqsubset Q_{2}$, and $Q_{3} \sqsubset Q_{4}$. Also, the return tuples of $Q 1$ that relate to the return tuples of $Q 3$ are identified (for example using the overlapping inference rule), and similarly for those between $Q 2$ and $Q 4$. If one of the following three conditions holds,

1. for each return tuple $t_{1}$ of $Q_{1}$ that does not relate to any return tuple of $Q_{3}, t_{1}$ is distinguishable from all return tuples of $Q_{4}$,

2. $Q_{4} \sqsubset Q_{3}$, or

3. $\left|Q_{3}\right|=\left|Q_{4}\right|$,

then $Q_{1}^{\prime} \sqsubset Q_{2}^{\prime}$, where $Q_{1}^{\prime}=\left(A S_{1} ; S C_{1} \wedge \neg S C_{3}\right)$, and $Q_{2}^{\prime}=\left(A S_{2} ; S C_{2} \wedge \neg S C_{4}\right)$. 
Figure 3(a) illustrates the case where condition (1) holds. Let $T_{1}$ be the set of return tuples of $Q_{1}$ that do not relate to any return tuple of $Q_{3}$, and $T_{2}$ be the set of return tuples of $Q_{2}$ that do not relate to any return tuple of $Q_{4}$. As $Q_{1} \sqsubset Q_{2}$ and $T_{1} \subset\left\{Q_{1}\right\}$, each tuple in $T_{1}$ relates to a return tuple of $Q_{2}$. Condition (1) says that each tuple in $T_{1}$ does not relate to any return tuple of $Q_{4}$. Hence, each tuple in $T_{1}$ relates to a tuple in $T_{2}$. Figure $3(\mathrm{~b})$ illustrates the case where condition (2) or (3) holds. Condition (2) or (3) implies that $Q_{3} \sqsubset Q_{4}$ and $Q_{4} \sqsubset Q_{3}$. By removing from $Q_{1}$ and $Q_{2}$ the "same" set of return tuples, we have $Q_{1}^{\prime} \sqsubset Q_{2}^{\prime}$. We further illustrate this by an example. Consider the following four queries,

$$
\begin{aligned}
Q_{1}= & (\text { Name; Department }=\text { 'Marketing' }) \\
Q_{2}= & (\text { Salary; Department }=\text { 'Marketing' } \vee \\
& \text { Office }=\text { '2nd Floor' }) \\
Q_{3}= & (\text { Name; Job }=\text { 'Secretary' }), \text { and } \\
Q_{4}= & (\text { Salary; Job }=\text { 'Secretary' }) .
\end{aligned}
$$

$Q_{1}$ returns two tuples (Alice) and (Bob). $Q_{2}$ returns three tuples (60K), (45K), and (65K). As $Q_{1} \sqsubset Q_{2}$, both Alice and Bob earn either $60 \mathrm{~K}, 45 \mathrm{~K}$, or $65 \mathrm{~K} . Q_{3}$ returns two tuples (Bob) and (Charles). $Q_{4}$ returns two tuples (45K) and (40K). As $S C_{3}=S C_{4}$, both Bob and Charles earn either $45 \mathrm{~K}$ or $40 \mathrm{~K}$. Now, we have $Q 1 \sqsubset Q 2, Q_{3} \sqsubset Q_{4}$, and $Q_{4} \sqsubset Q_{3},(B o b)$ is the only related tuple between $Q_{1}$ and $Q_{3}$ (assuming the employee names are unique), (45K) is the only related tuple between $Q_{2}$ and $Q_{4}$ (it is the only indistinguishable tuple between $Q_{2}$ and $Q_{4}$ ). By the complementary inference rule, $Q_{1}^{\prime} \sqsubset Q_{2}^{\prime}$, where

$$
\begin{aligned}
Q_{1}^{\prime}= & (\text { Name; Department }=\text { 'Marketing' } \wedge \\
& \text { Job } \neq \text { 'Secretary' }) \\
Q_{2}^{\prime}= & (\text { Salary; Department }=\text { 'Marketing' } \vee \\
& \text { Office }=\text { '2nd Floor' } \wedge \text { Job } \neq \text { 'Secretary' })
\end{aligned}
$$

$Q_{1}^{\prime}$ returns a single tuple (Alice), as it is the tuple returned by $Q_{1}$ but not by $Q_{3}$. $Q_{2}^{\prime}$ returns two tuples $(60 K)$ and $(65 K)$, as they are the tuples returned by $Q_{2}$ but not by $Q_{4}$. Therefore, the user can infer that Alice earns either $60 \mathrm{~K}$ or $65 \mathrm{~K}$.

\subsection{Functional Dependency Inference}

The functional dependency inference rule employs the functional dependencies among the attributes to perform inferences. It simulates the uses of functional dependencies in schema level inference detection systems.

Inference Rule 5 (Functional Dependency) Given that attribute $A_{1}$ functional determines attribute $A_{2}$, and there exists a tuple $t$, such that $t\left[A_{1}\right]=a_{1}$ and $t\left[A_{2}\right]=a_{2}$. If there is a tuple $t_{i}$, such that $t_{i}\left[A_{1}\right]=a_{1}$, then $t_{i}\left[A_{2}\right]=a_{2}$. The same applies when $A_{1}$ or $A_{2}$ is a composite attribute (that is, a group of attributes).

For example, if it is known that the attribute 'Department' functionally determines the attribute 'Office', and in particular the Marketing department is located on the 2nd Floor. Then, whenever a user knows a person who works in the Marketing department, the user knows the office of that person is located on the 2nd Floor. A similar rule exists for multivalue functional dependencies.

\subsection{Inference with Union Queries}

In this section, we discuss the use of a union of queries in inferences. Consider the following three queries,

$$
\begin{aligned}
& Q_{1}=(\text { Job; Age }<50 \wedge \text { Age }>40), \\
& Q_{2}=(\text { Job; Age }>45 \wedge \text { Age }<60), \text { and } \\
& Q_{3}=(\text { Job; Age }>30 \wedge \text { Age } \leq 45) .
\end{aligned}
$$

since the following implication holds,

$($ Age $<50 \wedge$ Age $>40) \rightarrow$

$(($ Age $>45 \wedge$ Age $<60) \vee($ Age $>30 \wedge$ Age $\leq 45))$, $Q_{1} \sqsubset\left(Q_{2} \cup Q_{3}\right)$ holds. The inference rules can still be applied by treating $\left(Q_{2} \cup Q_{3}\right)$ as a single user query. We call such a union of queries a 'union query'. In contrast, a user query is called a 'simple query'. If $Q_{u}$ is a union query that consists $Q_{i}, \ldots$, and $Q_{j}$, then $A S_{u}=\left(A S_{i} \cap \ldots \cap A S_{j}\right)$, and $S C_{u}=\left(S C_{i} \vee \ldots \vee S C_{j}\right)$. The applications of the unique characteristic and functional dependency inference rules on a union query are the same as their applications on the simple queries of the union query. Hence, we only consider the applications of the subsume, overlapping, and complementary inference rules on union queries.

Consider the applications of the subsume inference rule on union queries. Suppose $\left(Q_{2} \cup Q_{3}\right) \sqsubset Q_{1}$. This implies that $\left(Q_{2} \sqsubset Q_{1}\right.$ and $\left.Q_{3} \sqsubset Q_{1}\right)$. If the subsume inference rule is applicable due to $\left(Q_{2} \cup Q_{3}\right) \sqsubset Q_{1}$, then it is also applicable due to $\left(Q_{2} \sqsubset Q_{1}\right)$ and $\left(Q_{3} \sqsubset\right.$ $\left.Q_{1}\right)$. Hence, we do not need to consider the application of the subsume inference rule when the union query occurs on the left hand side of a ' $\square$ ' relation. Now, suppose $Q_{1} \sqsubset Q_{u}$, where $Q_{u}$ is a union query. The application of $S I 1$ on union queries is the same as when only simply queries are involved. To apply SI2, the user must has identified all the overlapping tuples among the simple queries of $Q_{u}$ that correspond to the return tuples of $Q_{1}$. The subsume inference rule can still be applied when the simple queries of $Q_{u}$ have no common projected attribute.

Consider the applications of the overlapping inference rule on union queries. Firstly, consider the application of $O I 1$. If $Q_{u} \sqsubset Q_{1}$ is involved, $\left|Q_{u}\right|$ must be 
known to the user. If $Q_{1} \sqsubset Q_{u}$ is involved, the user must has identified all the overlapping tuples among the simple queries of $Q_{u}$ that relate to the return tuples of $Q_{1}$. Now, consider the application of OI2. If $Q_{u} \sqsubset Q_{1}$ is involved, the user must has identified all the overlapping tuples among the simple queries of $Q_{u}$ that relate to the return tuples of $Q_{1}$. If $Q_{1} \sqsubset Q_{u}$ is involved, $\left|Q_{u}\right|$ must be known to the user. In either case, the attribute set of the union query cannot be empty.

To apply the complementary inference rule on union queries, the overlapping tuples of the simple queries in the union query must have been identified. Also the attribute set of the union query cannot be empty.

Finding all the eligible union queries that have the ' $\square$ ' relations with other queries is an NP-hard problem. This is because for each simple query $Q_{1}$, we need to find all union queries $Q_{u}$ such that $Q_{1} \sqsubset Q_{u}$ holds. This becomes the problem of finding a set of simple queries that together returns a set of tuples that covers another set of return tuples. If we can solve this problem, we can also solve the set-covering problem which is known to be an NP-complete problem [4]. We have developed a prototype to study the performance of the inference detection system in practice. It is discussed in Section 5.

\section{Implementation and Preliminary Re- sults}

We have developed a prototype of the inference detection system in about 4,000 lines of Perl code. We have implemented the subsume, unique characteristic, overlapping, and complementary inference rules. We run our experiments with randomly generated tables and user queries. Each table has $N_{a t t r}$ number of attributes, and $N_{\text {rec_num }}$ number of records. The primary key of the table is a single attribute. All attributes are of integer types. Each attribute value in the table is uniformly distributed between 0 and $\left(N_{\text {data_dist }} \times N_{\text {rec_num }}\right)$, where $0<N_{\text {data_dist }} \leq 1$. We also randomly generate $N_{\text {query_num }}$ number of user queries. Each query projects $N_{\text {proj }}$ number of attributes from the table. The selection criterion of each query is a conjunction of $N_{\text {cond }}$ number of conjuncts. Each conjunct is of the form ' $A_{i}$ op $a_{i}$ ', where $A_{i}$ is an attribute from the table, $o p$ is one of the relational operations $(>, \geq, \leq,<$, and $=)$, and $a_{i}$ is an attribute value. We only consider queries with the number of return tuples falls between 1 and $\left(N_{\text {set_size }} \times N_{\text {rec_num }}\right)$, where $0<N_{\text {set_size }} \leq 1$. We approximate the evaluation of a logical implication $C_{i} \rightarrow C_{j}$ by checking if the tuples selected by $C_{i}$ is also selected by $C_{j}$, and that the set of attributes appears in $C_{j}$ is a subset of those appear in $C_{i}$.

The preliminary results of running the inference detection system on a Sun SPARC 20 workstation are shown in Table 1-Table 4 . In each experiment, we run the inference detection system against 500 user queries. We have collected data about 1 ) the average number of seconds used to process one query; 2) the number of inferred queries generated; 3 ) the number of times the inference rules are applied; and 4) the ratio between the number of attribute values of those individual records that have been identified by the user (either by directly accessing them using a query or by inferences) and the total number of attribute values in the database. The ratio is denoted as "\% of DB revealed". For example, consider the two queries $Q_{1}$ and $Q_{2}$ in Section 4.1. These two queries together reveals that Alice is 35 years old, and she works in the Marketing department. Hence, the number of attribute values revealed to the user is 3 (namely Alice's name, Alice's age, and Alice's department). Note that although $Q_{2}$ returns two tuples, the user cannot determine whom these two tuples belong to; hence, they are not included as the attribute values that are revealed to the user. The total number of attribute values in the sample database is 24 (there are four records, each with 6 attribute values). Hence, the ' $\%$ of DB revealed' by $Q_{1}$ and $Q_{2}$ to the sample database is $(3 / 24) \times 100 \%$, or $12.5 \%$.

Table 1 shows the results for $N_{\text {rec_num }}=1000$, $N_{\text {set }_{s} i z e}=10 \%, N_{\text {proj }}=4, N_{\text {cond }}=3, N_{\text {query_num }}$ $=500, N_{\text {data_dist }}$ takes the values of $33 \%, 66 \%$, and $100 \%$, and $N_{a t t r}$ takes the values of 50,70 , and 90 . The number of tuples returned by each query is about 30 . It shows that the system performs better as $N_{a t t r}$ increases. This is because the larger the number of attributes in the table, the lesser the chance that the ' $\square$ ' relations hold among queries. Also, the system performs better when $N_{\text {data_dist }}$ decreases. The lower the distribution of the data, the more duplication of the data values, the lesser the chance a return tuple will be distinguishable from others, and hence the smaller number of occurrences of inferences. Table 2 shows similar results for $N_{\text {rec_num }}$ equals 10,000 . The number of tuples returned by each query is about 200 .

Table 3 shows the results for $N_{\text {rec_num }}=1000$, $N_{\text {data_dist }}=50 \%, N_{\text {attr }}=80, N_{\text {proj }}=4, N_{\text {cond }}=3$, and $N_{\text {query_num }}=500$, and $N_{\text {set_size }}$ takes the values of $10 \%, 20 \%, 30 \%, 40 \%$, and $50 \%$. It shows that the system performs better when $N_{\text {set_size }}$ decreases. This is because the more the number of records returned by the queries, the more the number of occurrences of inferences, and also the more the number of inferred queries being generated. 


\begin{tabular}{|c|c|c|c|c|c|}
\hline $\begin{array}{c}N_{\text {data_dist }} \\
(\%)\end{array}$ & $N_{\text {attr }}$ & $\begin{array}{c}\text { average query } \\
\text { processing time (sec) }\end{array}$ & $\begin{array}{c}\text { number of } \\
\text { inferred queries }\end{array}$ & $\begin{array}{c}\text { number of } \\
\text { inferences }\end{array}$ & $\begin{array}{c}\text { \% of DB } \\
\text { inferred }\end{array}$ \\
\hline \hline 33 & 50 & 1.990 & 40 & 3 & 6.94 \\
\hline 33 & 70 & 1.622 & 14 & 0 & 3.42 \\
\hline 33 & 90 & 1.532 & 7 & 0 & 3.06 \\
\hline 66 & 50 & 2.208 & 49 & 5 & 8.30 \\
\hline 66 & 70 & 1.632 & 18 & 0 & 3.45 \\
\hline 66 & 90 & 1.608 & 7 & 0 & 3.74 \\
\hline 100 & 50 & 2.386 & 50 & 11 & 8.79 \\
\hline 100 & 70 & 1.642 & 18 & 0 & 3.78 \\
\hline 100 & 90 & 1.650 & 9 & 0 & 3.95 \\
\hline
\end{tabular}

Table 1. $N_{\text {rec_num }}=1000, N_{\text {set_size }}=10 \%, N_{\text {proj }}=4, N_{\text {cond }}=3, N_{\text {query_num }}=500$.

\begin{tabular}{|c|c|c|c|c|c|}
\hline $\begin{array}{c}N_{\text {data_dist }} \\
(\%)\end{array}$ & $N_{\text {attr }}$ & $\begin{array}{c}\text { average query } \\
\text { processing time (sec) }\end{array}$ & $\begin{array}{c}\text { number of } \\
\text { inferred queries }\end{array}$ & $\begin{array}{c}\text { number of } \\
\text { inferences }\end{array}$ & $\begin{array}{c}\text { \% of DB } \\
\text { inferred }\end{array}$ \\
\hline \hline 33 & 50 & 3.876 & 53 & 14 & 6.12 \\
\hline 33 & 70 & 4.534 & 27 & 9 & 5.67 \\
\hline 33 & 90 & 2.484 & 13 & 0 & 1.82 \\
\hline 66 & 50 & 4.948 & 65 & 19 & 7.01 \\
\hline 66 & 70 & 5.534 & 35 & 9 & 6.13 \\
\hline 66 & 90 & 2.848 & 19 & 0 & 2.23 \\
\hline 100 & 50 & 6.160 & 87 & 99 & 7.45 \\
\hline 100 & 70 & 6.618 & 45 & 39 & 6.34 \\
\hline 100 & 90 & 3.002 & 19 & 0 & 2.29 \\
\hline
\end{tabular}

Table 2. $N_{\text {rec_num }}=10000, N_{\text {set_size }}=10 \%, N p r o j=4, N c o n d=3$.

\begin{tabular}{|c|c|c|c|c|}
\hline $\begin{array}{c}N_{\text {set_size }} \\
(\%)\end{array}$ & $\begin{array}{c}\text { average query } \\
\text { processing time (sec) }\end{array}$ & $\begin{array}{c}\text { number of } \\
\text { inferred queries }\end{array}$ & $\begin{array}{c}\text { number of } \\
\text { inferences }\end{array}$ & $\begin{array}{c}\text { \% of DB } \\
\text { inferred }\end{array}$ \\
\hline \hline 10 & 1.700 & 34 & 0 & 1.85 \\
\hline 20 & 1.890 & 23 & 0 & 5.77 \\
\hline 30 & 1.956 & 20 & 3 & 5.76 \\
\hline 40 & 2.152 & 22 & 3 & 8.08 \\
\hline 50 & 2.168 & 16 & 3 & 8.03 \\
\hline
\end{tabular}

Table 3. $N_{\text {rec_num }}=1000, N_{\text {data_dist }}=50 \%, N_{a t t r}=80, N_{\text {proj }}=4, N_{\text {cond }}=3, N_{\text {query_num }}=500$.

\begin{tabular}{|c|c|c|c|c|c|}
\hline$N_{\text {proj }}$ & $N_{\text {cond }}$ & $\begin{array}{c}\text { average query } \\
\text { processing time (sec) }\end{array}$ & $\begin{array}{c}\text { number of } \\
\text { inferred queries }\end{array}$ & $\begin{array}{c}\text { number of } \\
\text { inferences }\end{array}$ & $\begin{array}{c}\text { \% of DB } \\
\text { inferred }\end{array}$ \\
\hline \hline 4 & 3 & 1.700 & 34 & 0 & 1.85 \\
\hline 4 & 4 & 1.400 & 3 & 0 & 1.93 \\
\hline 4 & 5 & 1.452 & 3 & 0 & 2.76 \\
\hline 4 & 6 & 1.432 & 1 & 0 & 2.31 \\
\hline 3 & 3 & 1.346 & 5 & 0 & 2.22 \\
\hline 5 & 3 & 1.800 & 28 & 0 & 4.29 \\
\hline 6 & 3 & 2.556 & 68 & 0 & 7.30 \\
\hline
\end{tabular}

Table 4. $N_{\text {rec_num }}=1000, N_{\text {data_dist }}=50 \%, N_{\text {attr }}=80, N_{\text {set_size }}=10 \%$. 
Table 4 shows the results for $N_{\text {rec_num }}=1000$, $N_{\text {data_dist }}=50 \%, N_{\text {attr }}=80, N_{\text {set_size }}=10 \%, N_{\text {proj }}$ takes the values of $3,4,5$, and 6 while $N_{\text {cond }}$ is kept constant at 3 , and $N_{\text {cond }}$ takes the values of $3,4,5$, and 6 while $N_{\text {proj }}$ is kept constant at 4 . It shows that the system performs better when $N_{\text {proj }}$ decreases. This is because the more the number of attributes projected by the queries, the more overlapping among the queries, and hence the more number of inferences can occur. Also, the system performs better when $N_{\text {cond }}$ increases. This is because with a larger number of conjuncts in the selection criteria of the queries, there is lesser chance that the ' $\square$ ' relations hold among the queries, and hence the smaller number of occurrences of inferences.

In general, we expect to see the inference detection system performs better with the larger number of attributes in the table, the more duplication of attribute values in the database, the smaller number of records returned by queries, the smaller number of attributes projected by the queries, and the larger number of conjuncts in the selection criteria of the queries.

\section{Conclusions}

In this paper, we describe our effort in developing a data level inference detection system. We have identified five inference rules: subsume, unique characteristic, overlapping, complementary, and functional dependency inference rules. These rules are sound but they are not necessarily complete. The existence of these inference rules shows that simply using functional dependencies to detect inferences is inadequate. We have developed a prototype of the inference detection system using Perl on a Sun SPARC 20 workstation. The preliminary results show that the system on average takes seconds to process a query for a database of thousands of records.

Although in theory detecting inferences at data level is an NP-hard problem, in practice, there are cases where the use of such approach is practical. In particular, this is the case when there is a limited amount of overlapping among the return tuples of the queries. We can further improve the system performance using distributed computing techniques. For example, the inference rules can be applied to the queries in parallel.

Instead of using the inference detection system to detect if a user has accessed particular data, we can also employ it as an anomaly detection system. For example, when a user has inferred certain amount of data in the database, it is reported to the security officer so that closer monitor to user activities will be carried out.

Acknowledgements The research reported in this paper is supported by the National Security Agency University Research Program under Contract DOD MDA904-96-1-0117, and by the CIA Office of Research and Development under Contract 96F 154000000. The authors would like to acknowledge the helpful comments made by the reviewers of this paper.

\section{References}

[1] N. R. Adam and J. C. Wortmann. Security-control methods for statistical databases: A comparative study. ACM Computing Surveys, 21(4):515-556, December 1989.

[2] L. J. Binns. Inference through secondary path analysis. In B. M. Thuraisingham and C. E. Landwehr, editors, Database Security VI: Status and Prospects, pages 195-209. North-Holland, 1993.

[3] H. S. Delugach and T. H. Hinke. Wizard: A database inference analysis and detection system. IEEE Transactions on Data and Knowledge Engineering, 8(1):5666, 1996.

[4] M. R. Garey and D. S. Johnson. Computers and Intractability: A Guide to the Theory of NPCompleteness. W.H. Freeman, 1979.

[5] J. Hale and S. Shenoi. Catalytic inference analysis: Detection inference threats due to knowledge discovery. In Proceedings of the 1997 IEEE Symposium on Security and Privacy, pages 188-199. IEEE Computer Society Press, 1997.

[6] T. H. Hinke. Inference aggregation detection in database management systems. In Proceedings of the 1988 IEEE Symposium on Security and Privacy, pages 96-106. IEEE Computer Society Press, 1988.

[7] T. H. Hinke and H. S. Delugach. Aerie: An inference modeling and detection approach for databases. In B. M. Thuraisingham and C. E. Landwehr, editors, Database Security VI: Status and Prospects, pages 179-193. North-Holland, 1993.

[8] T. H. Hinke, H. S. Delugach, and A. Chandrasekhar. Layered knowledge chunks for database inference. In T. F. Keefe and C. E. Landwehr, editors, Database Security VII: Status and Prospects, pages 275-295. North-Holland, 1994.

[9] T. H. Hinke, H. S. Delugach, and R. Wolf. A framework for inference-directed data mining. In P. Samarati and R. S. Sandhu, editors, Database Security X: Status and Prospects, pages 229-239. Chapman and Hall, 1997.

[10] T. H. Hinke, H. S. Delugach, and R. P. Wolf. Iliad: An integrated laboratory for inference analysis and detection. In S. A. D. David L. Spooner and J. E. Dobson, editors, Database Security IX: Status and Prospects, pages $333-348,1995$ 
[11] T. F. Lunt. Aggregation and inference: Facts and fallacies. In Proceedings of the 1989 IEEE Symposium on Security and Privacy, pages 102-109. IEEE Computer Society Press, 1989.

[12] D. G. Marks. Inference in mls database systems. IEEE Transactions on Knowledge and Data Engineering, 8(1):46-55, February 1996.

[13] A. Motro, D. G. Marks, and S. Jajodia. Aggregation in relational databases: Controlled disclosure of sensitive imformation. In Proceedings of the Third European Symposium on Research in Computer Security, pages 431-445, November 1994.

[14] X. Qian, M. E. Stickel, P. D. Karp, T. F. Lunt, and T. D. Garvey. Detection and elimination of inference channels in multilevel relational database systems. In Proceedings of the 1993 IEEE Symposium on Security and Privacy, pages 196-205. IEEE Computer Society Press, 1993.

[15] M. E. Stickel. Elimination of inference channels by optimal upgrading. In Proceedings of the 1994 IEEE Symposium on Research in Security and Privacy, pages 168-174. IEEE Computer Society Press, 1994.

[16] M. E. Stickel, T. D. Garvey, T. F. Lunt, and $\mathrm{X}$. Qian. Inference channel detection and elimination in knowledge-based systems. Technical report, SRI International, Octobet 1994.

[17] T.-A. Su and G. Ozsoyoglu. Data dependencies and inference control in multilevel relational database systems. In Proceedings of the 1987 IEEE Symposium on Security and Privacy, pages 202-211. IEEE Computer Society Press, 1987.

[18] B. Thuraisingham. The use of conceptual structures for handling the inference problem. In C. E. Landwehr and S. Jajodia, editors, Database Security V: Status and Prospects, pages 333-362. Elsevier Science Pub. Co., 1992. 